Meta

Journal des traducteurs

Translators' Journal

\title{
Québec et Canada : entre l'unilinguisme et le bilinguisme politique
}

\section{Chantal Gagnon}

Volume 59, numéro 3, décembre 2014

Traduction et plurilinguisme officiel

Translation and Official Multilingualism

URI : https://id.erudit.org/iderudit/1028659ar

DOI : https://doi.org/10.7202/1028659ar

Aller au sommaire du numéro

Éditeur(s)

Les Presses de l’Université de Montréal

ISSN

0026-0452 (imprimé)

1492-1421 (numérique)

Découvrir la revue

Citer cet article

Gagnon, C. (2014). Québec et Canada : entre l'unilinguisme et le bilinguisme politique. Meta, 59(3), 598-619. https://doi.org/10.7202/1028659ar
Résumé de l'article

Le Canada est un pays officiellement bilingue, en ce sens que la communication qui émane de son gouvernement central se fait dans les deux langues officielles, l'anglais et le français. Cependant, les gouvernements des provinces canadiennes sont unilingues, à une exception près (la province du Nouveau-Brunswick est officiellement bilingue). Le contexte québécois est particulier puisque cette province est la seule dont la majorité s'exprime en français, d'où son statut de province unilingue française. Cependant, tant d'un point de vue politique que démographique, la population anglophone a toujours joué un rôle clé dans la société québécoise. La présente étude a pour objectif d'observer les différentes pratiques de traduction officielle du gouvernement du Canada et du gouvernement du Québec. Plus précisément, nous analyserons la traduction des discours de différents chefs politiques au provincial et au fédéral. Afin d'obtenir des résultats comparables, nous étudierons tout particulièrement les discours entourant un seul événement, soit le référendum québécois de 1995. Nos recherches précédentes ont démontré qu'au gouvernement fédéral, la traduction des discours politiques varie entre autres en fonction de l'époque et du premier ministre au pouvoir. Nous posons l'hypothèse qu'étant donné la longue tradition de traduction institutionnelle de certains partis politiques, les stratégies de traduction adoptées par ces partis seront plus efficaces que celles adoptées par les partis peu habitués à faire traduire leurs discours.
Ce document est protégé par la loi sur le droit d'auteur. L'utilisation des services d'Érudit (y compris la reproduction) est assujettie à sa politique d'utilisation que vous pouvez consulter en ligne.

https://apropos.erudit.org/fr/usagers/politique-dutilisation/ 


\title{
Québec et Canada: entre l'unilinguisme et le bilinguisme politique
}

\author{
CHANTAL GAGNON \\ Université de Montréal, Montréal, Canada \\ chantal.gagnon.4@umontreal.ca
}

\section{RÉSUMÉ}

Le Canada est un pays officiellement bilingue, en ce sens que la communication qui émane de son gouvernement central se fait dans les deux langues officielles, l'anglais et le français. Cependant, les gouvernements des provinces canadiennes sont unilingues, à une exception près (la province du Nouveau-Brunswick est officiellement bilingue). Le contexte québécois est particulier puisque cette province est la seule dont la majorité s'exprime en français, d'où son statut de province unilingue française. Cependant, tant d'un point de vue politique que démographique, la population anglophone a toujours joué un rôle clé dans la société québécoise. La présente étude a pour objectif d'observer les différentes pratiques de traduction officielle du gouvernement du Canada et du gouvernement du Québec. Plus précisément, nous analyserons la traduction des discours de différents chefs politiques au provincial et au fédéral. Afin d'obtenir des résultats comparables, nous étudierons tout particulièrement les discours entourant un seul événement, soit le référendum québécois de 1995. Nos recherches précédentes ont démontré qu'au gouvernement fédéral, la traduction des discours politiques varie entre autres en fonction de l'époque et du premier ministre au pouvoir. Nous posons l'hypothèse qu'étant donné la longue tradition de traduction institutionnelle de certains partis politiques, les stratégies de traduction adoptées par ces partis seront plus efficaces que celles adoptées par les partis peu habitués à faire traduire leurs discours.

\section{ABSTRACT}

Canada is an officially bilingual country, in that communications are produced by the central government in both official languages, English and French. However, the governments of the Canadian provinces are unilingual, with one exception (the province of New Brunswick is officially bilingual). The province of Quebec is special in that it is the only province whose majority population is Francophone, hence its unilingual French status. However, given the political and demographic context of the province, the Englishspeaking population of Quebec has always played a key role. This study focuses on the different practices of official translation by the Governments of Canada and Quebec. To ensure comparable results, it analyzes the translation of speeches made by various political leaders at the provincial and federal levels during the 1995 Quebec referendum. Our previous research has shown that, at the federal level, the translation of political speeches varies, depending on the historical context and the Prime Minister in power at the time, among other factors. We hypothesize that, given the long tradition of institutional translation characteristic of some political parties, their translation strategies are more effective than those of political parties that do not have a tradition of the institutional translation of speeches and are thus less familiar with what translation involves.

\section{MOTS CLÉS/KEYWORDS}

politique, bilinguisme, unilinguisme, discours, Canada, partis politiques politics, bilingualism, monolingualism, speeches, Canada, political parties 


\section{Introduction}

Le Canada est un pays officiellement bilingue, en ce sens que la communication qui émane de son gouvernement central se fait dans les deux langues officielles, l'anglais et le français. Cependant, les gouvernements des provinces et des territoires canadiens sont unilingues, à une exception près (la province du Nouveau-Brunswick est officiellement bilingue). Le contexte québécois est particulier puisque cette province est la seule dont la majorité s'exprime en français, d'où son statut de province unilingue française. Selon le modèle présenté par Reine Meylaerts des régimes linguistiques et traductionnels territoriaux (2011: 752s), le Canada et ses provinces correspondent à un régime d'unilinguisme institutionnel combiné avec un régime de multilinguisme institutionnel.

En septembre 2012, la nouvelle première ministre du Québec nommait JeanFrançois Lisée responsable des relations avec la communauté anglophone. Comme l'écrivait un participant anglophone à un forum sur Internet, il s'agit d'un «Minister of Anglo-affairs, so to speak ${ }^{1} »$. Jamais auparavant un gouvernement du Québec n'avait songé à nommer un délégué à la communauté anglophone. Si plusieurs ont apprécié le geste du nouveau gouvernement (dont Graham Fraser, le commissaire aux langues officielles du gouvernement fédéral canadien ${ }^{2}$ ), d'autres ont critiqué le choix du délégué (dont Don Macpherson, journaliste au quotidien montréalais The Gazette $^{3}$ ). D’autres encore ont déploré la formulation du mandat qui lui a été donné. C'est notamment le cas de l'Acadien Rino Morin-Rossignol, journaliste à l'Acadie nouvelle et ancien conseiller politique sur la question linguistique au NouveauBrunswick:

«Faites-leur sentir qu'ils constituent une richesse pour nous tous et qu'ils sont membres à part entière de la nation québécoise.» Lors de la présentation de son Conseil des ministres, le 19 septembre dernier, c'est ainsi que la première ministre du Québec, Pauline Marois, formulait le mandat qu'elle donnait à son ministre responsable de Montréal, Jean-François Lisée, afin de «tisser des relations étroites» avec ses citoyens anglophones.

Attention. La première ministre n’a pas dit: «Les anglophones du Québec constituent une richesse... », mais bien: «Faites-leur sentir qu'ils constituent une richesse... " C'est moi qui souligne. Parce qu'il y a une nuance énorme. Certes, l'intention exprimée par Mme Marois est louable, mais quelle gaucherie dans la manière de l'affirmer! Surtout de la part d'un premier ministre ${ }^{4}$. (2012: 17)

Comme l'expliquait l'écrivain Pierre Nepveu (2012) dans une lettre au journal Le Devoir ${ }^{5}$, certains partis politiques vivent une relation antagoniste avec la communauté anglophone du Québec. C'est peut-être ce qui explique l'absence d'une stratégie de traduction des discours politiques à l'échelle de la province québécoise, contrairement à ce qui existe au gouvernement fédéral du Canada. En effet, même si la Loi sur les langues officielles ne les oblige pas à le faire, les partis politiques du gouvernement fédéral font traduire un grand nombre de leurs discours politiques pour le grand public. Dans la province de Québec, la traduction de discours politiques se fait rare, malgré l'importance politique de la communauté anglophone. Il y a à cela une exception notable: tous les discours du budget à l'Assemblée nationale sont traduits vers l'anglais, depuis au moins $1997^{6}$. Il est fort probable, cependant, que les hommes d'affaires de Wall Street soient les premiers destinataires de ces discours, plutôt que la communauté d'expression anglaise du Québec. 
Les stratégies de traduction utilisées par les partis politiques ont-elles des répercussions sur la réception des discours dans les médias? Voilà une question qui, à notre connaissance, n'a jamais été abordée. Nos recherches précédentes (Gagnon: 2006a; 2006b; 2009) ont démontré qu'au gouvernement fédéral, la traduction des discours politiques varie entre autres en fonction de l'époque et du premier ministre au pouvoir. Nous posons l'hypothèse qu'étant donné la longue tradition de traduction institutionnelle de certains partis politiques, les stratégies de traduction adoptées par ces partis seront plus efficaces que celles adoptées par les partis peu habitués à faire traduire leurs discours. Le présent article investiguera cette problématique. Pour évaluer l'efficacité des stratégies de traduction, nous examinerons la réception médiatique des discours traduits de notre corpus. Plus précisément, nous analyserons la réaction des journalistes de la presse écrite aux discours politiques étudiés.

Le référendum de 1995 sur la souveraineté du Québec constitue un excellent point de départ pour lancer une recherche de type comparatiste sur les différentes stratégies de traduction des partis politiques. En effet, à cette occasion, les discours traduits présentés à la société québécoise ont été largement commentés par la gent journalistique de l'époque, dans les deux communautés linguistiques officielles. Deux éléments viendront tout particulièrement éclairer l'analyse: les commentaires des journalistes à propos des discours traduits et les choix de traduction effectués.

Pour les besoins de l'étude, quatre discours politiques seront analysés, provenant de quatre partis, soit le Parti libéral du Canada, le Parti libéral du Québec, le Bloc Québécois et le Parti Québécois. Voici la liste des discours qui constituent le corpus:

- le message à la nation de Jean Chrétien, premier ministre du Canada;

- la réponse à ce message par Lucien Bouchard, chef de l'opposition officielle au Canada;

- le discours de la victoire de Jean Chrétien;

- le discours de la victoire de Daniel Johnson, chef de l'opposition officielle au Québec;

- le discours de la défaite de Jacques Parizeau, premier ministre du Québec.

Ces discours ont été choisis pour leur caractère marquant au cours de la campagne référendaire. Tant la presse locale que la presse nationale ont analysé le contenu et la traduction de ces discours, notamment les quotidiens La Presse, Le Devoir, The Gazette, le Toronto Star et The Globe and Mail. En outre, ils sont représentatifs des luttes de pouvoir qui ont eu lieu au cours de la campagne référendaire. Enfin, les discours correspondent à un échange discursif, ce qui facilite l'étude comparative.

\section{Politiques de traduction des partis politiques à l'étude}

Afin de bien mettre en contexte les partis politiques à l'étude, nous allons, avant de passer à l'analyse interprétative, revoir les politiques de traduction des quatre partis. Nous utilisons les sites Web des partis pour illustrer leur politique de traduction respective. Nous avons eu recours au site d'archives Wayback Machine de l'organisme Internet Archive pour étudier l'historique des sites Web de chacun des partis. Fondé en 1996, Internet Archive est un organisme à but non lucratif dont l'objectif est de créer une bibliothèque de sites Internet, accessible gratuitement aux chercheurs, aux historiens, aux universitaires, aux personnes handicapées et au grand public ${ }^{7}$.

Puisque les archives d'Internet Archive ne sont pas exhaustives, il est difficile d'établir à quelle date les différents partis ont mis leur site en ligne pour la première fois. Selon nos recherches dans Internet Archive, le Parti libéral du Canada semble 
avoir son site depuis au moins $1996^{8}$, le Bloc Québécois semble avoir un site depuis au moins 19979. Quant aux sites du Parti Québécois et du Parti libéral du Québec, ils semblent exister depuis au moins $1998^{10}$ et 11 . Puisqu'il ne nous était pas possible d'avoir accès aux sites de 1995 (si, bien sûr, ces sites existaient), nous avons choisi les années 2000, 2003 et 2004 comme point de départ. Ces dates ont été retenues parce qu'elles correspondent aux années au cours desquelles les sites du Parti Québécois (PQ) et du Bloc Québécois (BQ) étaient multilingues; ces sites sont aujourd'hui unilingues. En étudiant les sites multilingues de ces deux partis, nous serons en mesure d'évaluer leur positionnement par rapport aux différentes communautés linguistiques au Québec. Pour faciliter la comparaison, nous avons également choisi les premières années du second millénaire pour l'étude du site du Parti libéral du Canada (PLC) et du Parti libéral du Québec (PLQ). Selon les archives consultées, les sites du PLC et du PLQ ont toujours été bilingues.

\subsection{Parti Québécois (PQ)}

Fondé en 1968 par un ancien ministre du Parti libéral, René Lévesque, le Parti Québécois œuvre sur la scène provinciale et il milite pour la souveraineté du Québec. C'est à l'initiative du parti, au moment où il était au pouvoir, que le référendum de 1995 a été organisé. Il s'agissait du deuxième référendum sur la souveraineté, le premier ayant eu lieu le 20 mai 1980.

En de rares occasions, le chef du Parti Québécois prononce des discours en anglais pour la communauté anglophone du Québec, pour le Canada anglais ou à l'étranger. On pense, par exemple, à deux discours de René Lévesque, comme "Québec: A Good Neighbour in Transition» prononcé à l'Economic Club de New York le 25 janvier 1977, ou à "Quebecers are Quebecois", prononcé le 23 mars 1980 à Montréal. À une certaine époque, René Lévesque avait son antenne dans le monde anglophone; Évelyn Dumas traduisait ses discours, mais Lévesque mettait aussi la main à la pâte pour ses traductions vers l'anglais (Godin 2001; Lisée 1990). Dans l'ensemble, les anciens chefs du Parti Québécois s'exprimaient extrêmement bien dans la langue de Shakespeare et ils répondaient en anglais aux questions posées par les journalistes d'expression anglaise. Cependant, les allocutions politiques du Parti Québécois se font généralement en langue française. Il appartient aux journalistes anglophones de traduire vers l'anglais les extraits qui les intéressent (voir Gagnon 2012).

Sous la direction du chef Bernard Landry, puis sous celle d'André Boisclair, le site Web du parti était offert en français, en anglais, en espagnol et en portugais (voir Figure 1). Contrairement au site de son grand rival, le Parti libéral du Québec, le Parti Québécois offrait alors son site en quatre langues plutôt qu'en deux langues. Sur la page d'accueil péquiste, l'anglais (et sa communauté) n'avait pas de statut spécial: il s'agissait d'une langue parmi d'autres. Par ailleurs, c'est le mot russe «Другие языки», qui veut dire «autres langues », qui annonçait la présence de pages rédigées en d'autres langues que le français. Ainsi, aucun mot de langue anglaise ne venait rompre l'harmonie française de la page d'accueil du Parti Québécois. Cette situation montre à quel point la relation entre le Parti Québécois et la langue anglaise est complexe. 
FIGURE $1^{12}$

Extrait d'un site Web du Parti Québécois (2003)

\begin{tabular}{|c|c|c|}
\hline wwwepqorg & \multirow{2}{*}{\multicolumn{2}{|c|}{$\begin{array}{l}\text { Actualité Le Parti Souveraineté Appuyez-nous Boutique } \\
\text { Parti Québécois }\end{array}$}} \\
\hline \multirow{10}{*}{$\begin{array}{l}\text { Mediatheque } \\
>\text { Vidéo } \\
>\text { Audio } \\
>\text { Documents }\end{array}$} & & \\
\hline & \multirow{3}{*}{$\begin{array}{l}\text { Bienvenue! } \\
\text { Le Parti Québécois est une } \\
\text { formation politique dont la } \\
\text { principale raison d'être est de } \\
\text { réaliser, démocratiquement, la } \\
\text { souveraineté du Québec. Son } \\
\text { programme est de tendance } \\
\text { social-démocrate. }\end{array}$} & Point de vue \\
\hline & & \multirow{2}{*}{ 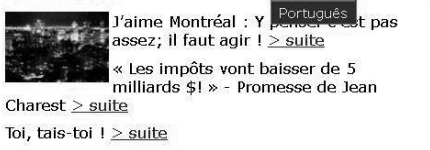 } \\
\hline & & \\
\hline & Souveraineté & $>$ Voir tous les Point de vue \\
\hline & Un état souverain vote & Nouvelles et communiqués \\
\hline & $\begin{array}{l}\text { concernent ; prélève lui-même } \\
\text { tous ses impôts ; signe en son }\end{array}$ & $\begin{array}{l}\text { Le } 6 \text { décembre } 1989 \text { : Se souvenir pour agir contre } \\
\text { la violence faite aux femmes } \geq \text { suite }\end{array}$ \\
\hline & $\begin{array}{l}\text { propre nom tous ses traités } \\
\text { internationaux. }\end{array}$ & \multirow{2}{*}{$\begin{array}{l}\text { Commission parlementaire sur le financement des } \\
\text { universités : Le ministre de l'éducation remet tout } \\
\text { en question } \geq \text { suite }\end{array}$} \\
\hline & Social-démocratie & \\
\hline & $\begin{array}{l}\text { Par opposition aux courants } \\
\text { néolibéraux ou conseryateurs, la } \\
\text { sociale-démocratie préconise }\end{array}$ & $\begin{array}{l}\text { Bernard Landry participe à l'inauguration officielle } \\
\text { de la permanence du Parti Québécois dans la } \\
\text { capitale nationale }>\text { suite }\end{array}$ \\
\hline
\end{tabular}

Avec l'arrivée de Pauline Marois au PQ en 2007, le parti est revenu à un site unilingue. Avec Marois à sa tête, le Parti Québécois a pris le pouvoir du gouvernement du Québec en 2012. Pour Lysiane Gagnon,

l'insensibilité de Mme Marois envers la minorité anglaise découle peut-être en partie du fait qu'elle est le premier leader péquiste à ne pas parler couramment anglais, contrairement à ses prédécesseurs qui le parlaient tous très bien, et certains à la perfection (Lévesque, Johnson et Parizeau ${ }^{13}$. (2012: 17)

Il existe peut-être un lien entre ces éléments et le retour à l'unilinguisme sur le site du Parti Québécois. Cela dit, comme nous l'avons expliqué en introduction, Pauline Marois est la seule première ministre de l'histoire à avoir confié officiellement le dossier de la communauté anglophone à un ministre. De ce point de vue, le terme «insensibilité» utilisé par la journaliste pour décrire l'attitude de la première ministre ne fait pas l'unanimité.

Même lorsque le site du Parti Québécois était quadrilingue, il y avait peu de contenu en anglais, en portugais et en espagnol. Par ailleurs, le quadrilinguisme du site ne semblait pas se refléter dans les pratiques au quotidien du PQ: les discours émanant du parti étaient généralement offerts en français seulement. De tout cela, on conclut que le Parti Québécois a une tradition de bilinguisme très occasionnel. Les conseillers politiques du chef du parti sont souvent habilités à s'exprimer en anglais, mais les politiciens du parti et leur entourage ne sont pas nécessairement appelés à le faire au quotidien.

\subsection{Bloc Québécois (BQ)}

Fondé en 1991 par Lucien Bouchard, le Bloc Québécois est lui aussi un parti souverainiste, mais il siège à la Chambre des communes du gouvernement fédéral. Contrairement aux autres partis fédéraux, il est établi exclusivement dans la province de Québec. Le 25 octobre 1993, le parti a été élu à titre d'opposition officielle de la Chambre des communes du Canada. Pour Scott Piroth (2012), l'arrivée du Bloc Québécois a contribué à améliorer le statut du français à la Chambre des communes. Le chercheur explique qu'au Canada, le nombre de comtés politiques où le français tient une place importante atteint de $24 \%$ à $28 \%$ du nombre total des comtés. Ainsi, 
lors de la période des questions au Parlement canadien, on pourrait s'attendre à ce que le français occupe un niveau qui se rapproche de ce pourcentage. Or, avant 1993, le français n'a jamais excédé $13 \%$ de la période des questions. Depuis l'arrivée du Bloc Québécois, le pourcentage de français utilisé lors de la période de questions s'est accentué de façon draconienne, dépassant même le pourcentage de comtés fédéraux où l'on s'exprime en français (Piroth, 2012: 167). Pour la journaliste Chantal Hébert $\left(2012^{14}\right)$, en forçant l'usage du français au Parlement fédéral, les députés du Bloc Québécois ont contribué à une révolution culturelle au sein de la Chambre des communes.

Parce qu'il œuvre sur la scène fédérale, le Bloc Québécois est constamment en présence de l'anglais: à la Chambre des communes, il y a une majorité de députés anglophones et la ville d'Ottawa, où est située la Chambre, a une population majoritairement anglophone. De plus, en campagne électorale, on s'attend à ce que le chef du parti participe au débat des chefs en anglais et à ce qu'il réponde en anglais aux questions des journalistes anglophones. Le Bloc Québécois est par conséquent un parti où il y a une tradition de bilinguisme occasionnel: il y existe une certaine expertise à propos de la rédaction et de la traduction en langue anglaise.

Du point de vue langagier, le site Web du parti a subi de grandes transformations avec les années. Au départ, le site était généralement trilingue, avec des documents surtout en français, mais aussi en anglais et en espagnol (voir la Figure 2 pour un exemple représentatif). Cependant, vers le début des années 2000, il s'est transformé en site unilingue français, forme qu'il a encore à ce jour.

FIGURE $2^{15}$

Extrait d'un site Web du Bloc Québécois (2000)

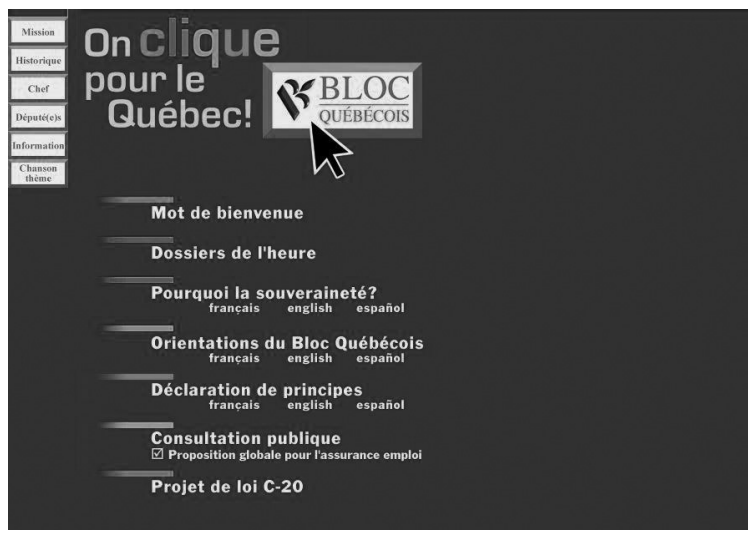

\subsection{Parti libéral du Canada (PLC)}

Fondé en 1867, le Parti libéral du Canada est le plus ancien au pays ${ }^{16}$. Selon l'École de politique appliquée de l'Université de Sherbrooke,

[1]es politiques [du Parti libéral du Canada] sont marquées par une volonté de promouvoir le progrès social par l'interventionnisme de l'État et par un désir de concilier les deux principales communautés linguistiques du pays (francophone et anglophone ${ }^{17}$. (École de politique appliquée de l'Université de Sherbrooke, 2013) 
Étant donné les choix politiques de ce parti, il y existe une forte tradition de bilinguisme et de traduction. Le parti est fier d'avoir participé à l'élaboration des politiques linguistiques officielles du Canada. Tous les grands discours de ses chefs sont traduits depuis au moins la mise en place de la Loi sur les langues officielles en 1969. Depuis le passage de Pierre Elliott Trudeau à la tête du parti, en 1968, tous les chefs du parti ont été bilingues, c'est-à-dire qu'ils étaient en mesure de répondre en français et en anglais aux médias et de faire des discours dans les deux langues officielles. De plus, comme l'explique le journaliste Raymond Giroux $\left(2011^{18}\right)$, il existe une tradition au PLC à propos de la langue maternelle du chef: "Rappelons que, hasard ou pas, les militants libéraux respectent depuis toujours la tradition de l'alternance entre anglophones et francophones pour diriger le parti.» En outre, en 2006, l'un des députés du PLC avait affirmé, au moment d'une course à la direction du parti, que les candidats unilingues perdaient leur temps en se présentant dans la course $^{19}$.

$\mathrm{Au}$ PLC, la volonté de bilinguisme dépasse le seul choix du chef. Par exemple, les communiqués de presse du parti sont bilingues, ainsi que le site Web du parti (voir figures 3 et 4). En fait, le site Web du PLC va au-delà de la simple traduction du texte: il adapte le texte des images d'une version linguistique à l'autre. Dans les figures ci-dessous, on voit à droite l'image d'un journal qui présente un texte différent dans les deux versions linguistiques, soit «The Liberal Press» en anglais et «La Presse libérale» en français. Ce souci du détail dans la traduction illustre à quel point le bilinguisme est ancré dans les pratiques du Parti libéral du Canada.

FIGURE $3^{20}$

Extrait d'un site Web du Parti libéral du Canada - version anglaise (2000)

\begin{tabular}{|c|c|c|}
\hline & FRANCAIS & HOME \\
\hline \multirow[b]{2}{*}{$\begin{array}{r}\text { THE TEAM } \\
\text { PARTY STRUCTURE } \\
\text { COMMISSIONS }\end{array}$} & \multirow[t]{4}{*}{ 웜 } & $\begin{array}{r}\text { WEEK OF JANUARY } 17 \\
2000\end{array}$ \\
\hline & & $\begin{array}{l}\text { Read All About If ! } \\
\text { The uberal Times in PCF } \\
\text { pol times }\end{array}$ \\
\hline \multirow{2}{*}{\multicolumn{2}{|c|}{$\begin{array}{l}\text { JOIN THE PARTY } \\
\text { FEEDBACK }\end{array}$}} & \\
\hline & & \\
\hline 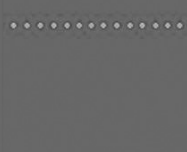 & $\begin{array}{l}\text { The Final Job Numbers for } 1999 \\
\text { Statistics Canada reported that } 1999 \text { was a very good } \\
\text { year for Canadians looking for work with } 355,000 \text { net } \\
\text { new jobs being created. It also reported that full-time } \\
\text { jobs increased by } 438,000 \text { over the year. }\end{array}$ & New APC Website \\
\hline
\end{tabular}


FIGURE $4^{21}$

Extrait d'un site Web du Parti libéral du Canada - version française (2000)

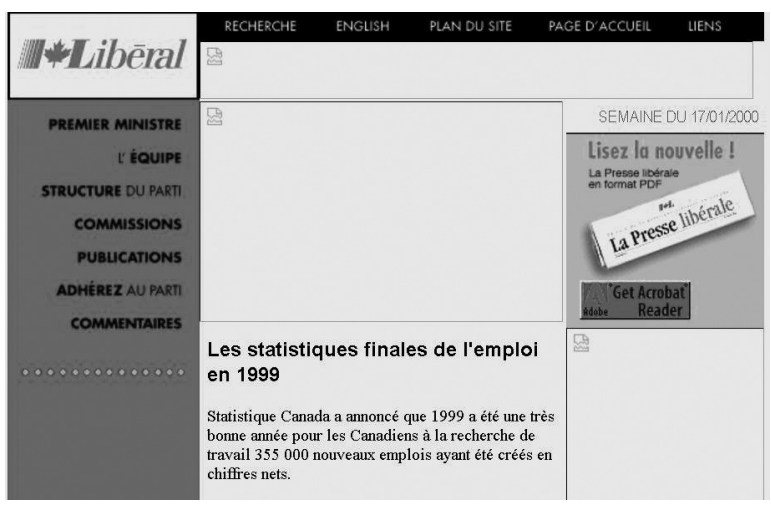

\subsection{Parti libéral du Québec (PLQ)}

Le Parti libéral du Québec (PLQ) est le plus ancien parti de la «Belle Province». Il partage une vision commune avec le Parti libéral du Canada. Le vote de la communauté d'expression anglaise (8\% de la population québécoise) est traditionnellement acquis au PLQ ${ }^{22}$ et le chef de ce parti a toujours été en mesure de bien s'exprimer en anglais. D’ailleurs, le président du PLQ affirmait en 2007 que son parti était le seul à courtiser l'électorat anglophone ${ }^{23}$. Il est vrai que, d'après nos recherches, le site du PLQ a toujours été bilingue. Cependant, le bilinguisme du PLQ ne ressemble pas au bilinguisme du PLC, son parti frère. En effet, le contenu du site du PLC est intégralement traduit, alors qu'au PLQ, seule une partie du contenu est traduite. En naviguant sur le site de ce dernier, il arrive qu'en cliquant sur l'hyperlien «English», on obtienne une page rédigée en français et en anglais, avec la mention «(All of this page is not available in English)». Pourtant, dans le même site, les électeurs d'expression française ne sont jamais exposés à du contenu de langue anglaise (voir figures 5 et 6 ci-dessous).

FIGURE $5^{24}$

Extrait d'un site Web du Parti libéral du Québec - version anglaise (2004)

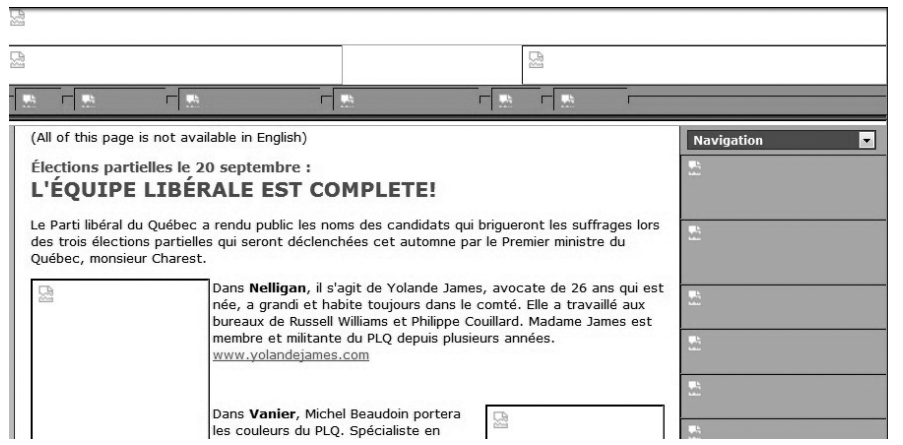


FIGURE $6^{25}$

Extrait d'un site Web du Parti libéral du Québec - version française (2004)

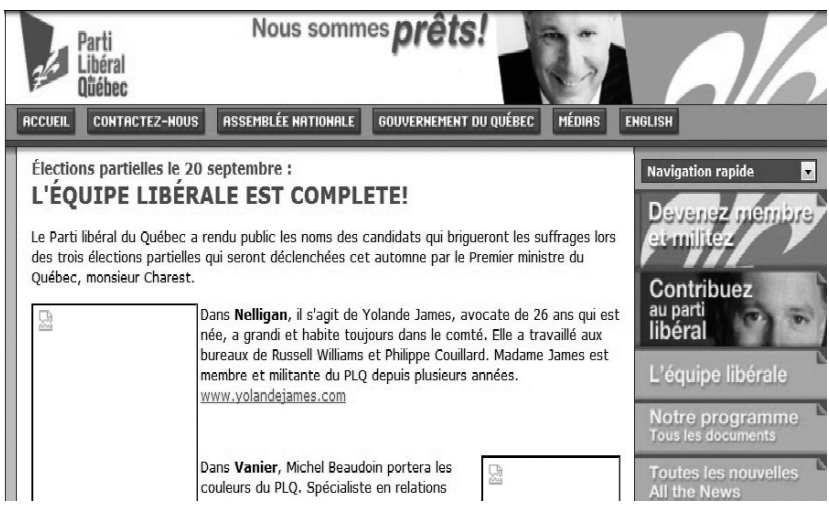

\subsection{Conclusion provisoire}

Résumons-nous. Pendant les quelques années où les sites Web du BQ et du PQ ont offert des documents en anglais aux internautes, ils l'ont fait à titre de sites multilingues. Chez les deux partis souverainistes, le bilinguisme ne semble pas être la voie admissible, politiquement parlant, pour atteindre l'électorat d'expression anglaise du Québec. Le fait que ces deux partis militent activement pour la défense de la langue française au Québec (et contre une éventuelle anglicisation de la province) explique peut-être leur politique de traduction. Par ailleurs, le choix même des langues de traduction invite à penser que ce ne sont pas seulement les communautés anglophones, hispanophones et lusophones qui sont ciblées. Logiquement, on s'attendrait à ce que ces deux partis s'adressent aux communautés immigrantes les plus importantes du Québec. Or, selon Valérie Amireault (2004: 58), «[a]u Québec, la langue maternelle la plus répandue chez les immigrants demeure, en 2001, l'italien, suivie de la langue arabe en deuxième place. L'arabe devance ainsi l'espagnol qui était deuxième en 1996.»Cela dit, si l'espagnol et le portugais n'occupent pas nécessairement une place prépondérante dans la province québécoise, ces langues sont cependant incontournables en contexte continental: l'espagnol, l'anglais, le portugais et le français sont, en ordre d'importance, les langues les plus parlées au sein des Amériques. C'est dire que l'image «non francophone» des partis souverainistes importe autant sinon plus à l'échelle mondiale qu'à l'échelle locale. Par l'intermédiaire de leur site Web, le Parti Québécois et le Bloc Québécois semblent s'adresser aux communautés linguistiques de l'Amérique du Nord et de l'Amérique du Sud, aux États-Unis, au Mexique et au Brésil, par exemple. Ces choix linguistiques ont pour effet de minimiser l'importance de la communauté d'expression anglaise du Québec. D’ailleurs, un courriel du coordonnateur du portail du gouvernement du Québec en 2002 appuie notre interprétation:

Vous avez certes raison en mentionnant que la communauté italophone au Québec est plus importante que la communauté espagnophone [sic]. Cependant, d'autres critères ont présidé au choix de cette troisième langue dont celui, purement mathématique, de l'importance de telle ou telle langue au niveau mondial ${ }^{26}$. (Bélisle 2002) 
En 2002, le portail du gouvernement présentait lui aussi des traductions anglaises et espagnoles. Il faut savoir qu'à l'époque, le gouvernement était dirigé par le Parti Québécois. Sous la gouvernance du Parti libéral du Québec, entre 2003 et 2012, Portail Québec a laissé tomber l'espagnol; le site est bilingue depuis au moins décembre 2008. L’utilisation du multilinguisme pour évacuer les problèmes politiques du bilinguisme n'est pas propre à la province du Québec. En Belgique, par exemple, dans la Région flamande, le ministre de l'Intérieur, Marino Keulen, a fait paraître en 2005 une circulaire qui balisait l'utilisation de certaines langues dans les communications destinées aux touristes ${ }^{27}$. Le bilinguisme néerlandais-français est formellement interdit:

Dans les communes qui sont considérées comme centres touristiques (par exemple les communes côtières), les conseils communaux peuvent décider que les avis et communications destinés aux touristes soient rédigés dans au moins trois langues. L'article 11, $\$ 3$, des L.L.C. est très clair à ce sujet: il doit s'agir de trois langues au moins, dont le néerlandais, que les conseils communaux peuvent définir librement. Il n'est donc pas possible que, dans les communes concernées, les avis et les communications ne soient rédigés qu’en néerlandais et en français ${ }^{28}$. (2005: 6; nous soulignons)

Pour Reine Meylaerts, les luttes linguistiques font partie intégrante de l'histoire belge (2009: 8). Comme le Québec et le Canada, la Belgique est donc aux prises avec des conflits linguistiques importants. En fait, on constate avec De Coster que tout clivage linguistique «révèl[e] le souci premier de préserver une culture, et, partant, une identité, dans la mesure où la langue en représente un élément incontestablement important» (2007: 13). Cependant, les enjeux politiques associés à la langue ne se retrouvent pas exclusivement dans les pays dits bilingues ou multilingues; ils sont aussi le fait de pays dits unilingues, comme les États-Unis d'Amérique (Meylaerts 2009: 8). Dans le cas qui nous intéresse, toutefois, la problématique va au-delà de la planification en matière de traduction institutionnelle pour les besoins d'une communauté linguistique donnée. Car pour les partis politiques canadiens et québécois, il ne s'agit pas tant de répondre aux besoins d'une communauté linguistique, mais plutôt de répondre aux besoins d'une communauté d'électeurs. La politique de traduction des partis n'est donc pas dictée par les droits éventuels des communautés, mais par la préférence d'une catégorie d'électeurs. C'est ce qui explique le bilinguisme systématique du Parti libéral du Canada, le bilinguisme pragmatique du Parti libéral du Québec et le bilinguisme occasionnel, voire très occasionnel, du Bloc Québécois et du Parti Québécois. Au PLC et au PLQ, les électeurs appartiennent aux deux communautés linguistiques, alors qu'au Bloc et au Parti Québécois, ils appartiennent essentiellement à la communauté francophone.

La prochaine section portera sur la traduction et la réception de cinq discours politiques prononcés lors du référendum de 1995. Cet examen nous donnera l'occasion de vérifier s'il existe, chez les partis politiques à l'étude, des liens entre leurs politiques de traduction et l'efficacité de leurs stratégies de traduction.

\section{Traduction et réception des discours à l'étude}

En 1995, cinq discours ont marqué les esprits par leur force et leur contenu. Il s'agit de deux messages télévisés prononcés le 25 octobre 1995 par Jean Chrétien, premier ministre du Canada, et Lucien Bouchard, chef de l'opposition officielle à la Chambre des communes. Cinq jours plus tard, le 30 octobre 1995, au soir du référendum, trois 
discours ont eux aussi retenu l'attention des médias, soit ceux de Jean Chrétien, de Jacques Parizeau, premier ministre du Québec et de Daniel Johnson, chef de l'opposition officielle du gouvernement québécois. Soulignons que Lucien Bouchard était le chef du Bloc Québécois, alors que Jacques Parizeau était le chef du Parti Québécois et président du Comité pour le OUI. Quant à Jean Chrétien, il était le chef du Parti libéral du Canada. Daniel Johnson était chef du PLQ et président de la Coalition du NON. Nous porterons tout particulièrement attention à la réception de ces discours et de leur traduction dans les médias. En examinant la réaction de la presse écrite, nous serons en bonne position pour évaluer l'efficacité des stratégies de traduction des partis étudiés.

\subsection{Les deux discours prononcés avant le référendum de 1995}

Les discours de Chrétien et de Bouchard ont été prononcés dans les deux langues officielles, d'un océan à l'autre. Ces discours appartiennent à une catégorie particulière, intitulée «message à la nation». Ce type de discours fait partie d'une tradition politique qui permet aux chefs politiques les plus importants du gouvernement fédéral canadien de s'adresser à la population. Voici un extrait de la Politique de programmation de CBC/Radio-Canada, le télédiffuseur public:

Dans l'exercice du pouvoir exécutif, prévu par le système parlementaire du Canada, le gouvernement doit avoir accès au service national de radiodiffusion quand il veut s'adresser d'urgence à la population, l'informer sur des questions d'actualité d'importance nationale et solliciter sa coopération. [...] La Société programme généralement ces émissions aux heures de grande écoute, dans le cadre de la programmation régulière des chaînes anglaises et françaises de radio et de télévision ${ }^{29}$. (CBC/Radio-Canada 1995)

S’il n'est pas prévu dans cette politique (ni dans la loi) que le chef de l'opposition ait droit de réplique, traditionnellement, le premier ministre le lui accorde. C'est ce qui s'est produit en 1995. Le message à la nation est généralement disséqué, analysé et critiqué par les médias de la télévision et de la presse écrite.

Dans les deux discours, on retrouvait des écarts de traduction. Un écart de traduction est généralement lié aux différences linguistiques qui existent entre un texte original et sa traduction. Dans certains cas cependant, l'écart peut avoir une fonction idéologique. Il s'agit alors d'une stratégie de traduction, comme l'ont montré nos travaux précédents (voir Gagnon 2009). Dans le discours de Jean Chrétien, par exemple, le discours en français s'adressait principalement aux Franco-Québécois, même si le message à la nation était aussi diffusé chez les populations francophones à l'extérieur du Québec. En anglais, le discours s'adressait à tous les Canadiens (voir Gagnon 2006b):

(1) a. Pour la première fois de mon mandat de premier ministre, j'ai invoqué une procédure exceptionnelle pour m'adresser à vous ce soir.

b. For the first time in my mandate as Prime Minister, I have asked to speak directly to Canadians tonight.

(Canada, Premier ministre 1995a: 1, voir annexe; nous soulignons)

(2) a. Nous, Québécois, ne serions plus canadiens et n’aurions plus droit aux privilèges attachés à la citoyenneté canadienne [...]. 
b. Where Quebecers would no longer enjoy the rights and privileges associated with Canadian citizenship.

(Canada, Premier ministre 1995a: 1, voir annexe; nous soulignons)

Alors que les extraits en français sont à la deuxième ou à la première personne du pluriel, l'anglais est à la troisième personne du pluriel. Il est clair que le destinataire du discours change d'une version linguistique à l'autre. On remarque aussi qu'en français, le premier ministre mentionne qu'avec un "OUI», les Québécois «ne seraient plus Canadiens». On peut penser que dans la version française, le premier ministre fait allusion à l'identité canadienne; l'extrait anglais ne fait pas cette nuance. Cependant, on retrouve cette idée plus loin dans le texte anglais du discours:

(3) a. Ils ne devraient pas prendre un risque pareil.

b. Anyone who really wants to remain a Canadian should think twice before taking such a dangerous risk.

(Canada, Premier ministre 1995a: 1, voir annexe; nous soulignons)

Ici, l'énoncé «Quiconque veut réellement rester canadien» n’apparaît qu'en anglais. Il existe une différence de degré entre le positionnement identitaire de l'exemple (2) et celui de l'exemple (3). Ainsi, dans la version française de l'exemple (2), on présente le fait de ne plus être canadien comme une perte, mais dans l'exemple (3), on sous-entend en plus qu'il y a des Québécois qui désirent rester canadiens. L'attachement au Canada semble légèrement plus fort en anglais. Par ailleurs, dans l'exemple (2), l'anglais associe la citoyenneté au verbe positif «enjoy», alors qu'en français, la citoyenneté n'est associée qu'à un avantage, avec la formule "avoir droit». Les écarts repérés dans la traduction ciblent des mots ou des expressions; en ce sens, il s'agit de micro-écarts, c'est-à-dire des écarts qui transforment le texte à petite échelle. Par exemple, selon la taxonomie modifiée de Chesterman (Gagnon 2006a; 2009; suivant Chesterman 1997), les extraits (1), (2) et (3) touchent légèrement à la cohésion, ainsi qu'à certains renseignements du texte.

On peut en dire autant du discours de Lucien Bouchard, à la différence près qu'il s'agit d'écarts à la fois micro-textuels et macro-textuels. Ainsi, plusieurs des écarts repérés sont de l'ordre de la transrévision et de la réécriture bilingue (Gagnon 2006a; 2009; suivant Chesterman 1997) et ils transforment tout le texte. Ici, le terme «transrévision» est défini comme un processus par lequel un texte subit des transformations substantielles en cours de traduction; ces transformations répondent à de nouveaux critères, notamment linguistiques et culturels. Dans le discours de Bouchard, les différences entre le texte anglais et le texte français étaient si apparentes qu'au lendemain du discours, le quotidien torontois The Globe and Mail (1995a; voir annexe) a fait paraître sur la même page le texte officiel anglais de Lucien Bouchard, ainsi qu'une traduction maison de la version française (Gagnon 2013). Voici un exemple représentatif des écarts micro-textuels entre la version française et la traduction officielle:

(4) a. Arrachons ces étiquettes de fédéralistes et de souverainistes, reformons nos rangs et mettons fin à ces divisions qui nous affaiblissent. L'appareil fédéral n'a été fort que de nos faiblesses. Reconnaissons-nous pour ce que nous sommes, un peuple, un peuple vivant, fier, chaleureux et confiant.

(Le Point 1995; voir annexe; nous soulignons) 
b. Let us gather together all of our energies as federalists or as sovereignists. Let us Quebeckers recognize ourself [sic] for what we are, a people, a vibrant country - proud, welcoming and confident.

(The Globe and Mail 1995b: A16; voir annexe; nous soulignons)

Les écarts présentés ici appartiennent aux catégories de l'adaptation et de la modification de renseignements (Gagnon 2006a; 2009; suivant Chesterman 1997). Ces écarts entraînent des divergences importantes dans le message envoyé. Par exemple, le texte français fait allusion à des divisions qui minent les Québécois, alors que l'anglais invite au rassemblement des forces fédéralistes et souverainistes.

La modification de renseignements est fréquemment utilisée dans la traduction officielle anglaise, comme en fait foi cet autre extrait:

(5) a. Il serait gênant de demander à nouveau au Canada anglais de nous reconnaître comme peuple. Tout cela est fini. Nous sommes au-delà des supplications. De toute façon, elles nous ont menés nulle part. Toutes nos demandes se sont brisées sur des nons à répétition.

(Le Point 1995; voir annexe; nous soulignons)

b. It is demeaning to ask English Canada to recognise us as a people. All that is finished. We have gone beyond entreaties.

(The Globe and Mail 1995b: A16; voir annexe)

Ici, la version française est plus précise et plus détaillée. On explique notamment la raison pour laquelle les supplications ne font plus partie des stratégies envisagées par les Québécois pour se faire reconnaître comme peuple.

Les écarts macro-textuels étant plus longs, nous n'en montrerons qu'un. Il s'agit d'un cas de transrévision, où un extrait en français ne se retrouve pas dans la version officielle anglaise.

(6) Qu'est-ce donc qui, dans l'imminence de la décision, pousse $\mathrm{M}$. Chrétien à mettre sa stratégie et ses discours au rancart? La réponse est simple: c'est la possibilité de plus en plus sérieuse d'un OUI. Autrement dit, ce n'est pas un vent de changement qui passe sur Ottawa, mais un vent de panique. Voilà qui nous donne un avantgoût de l'effet d'un OUI. Si la seule appréhension d'un OUI, simplement possible, peut ébranler à ce point l'obstination d'Ottawa, songeons à la force politique que nous donnera un OUI exprimé, le OUI d'un peuple, le OUI d'une solidarité.

(Le Point 1995, voir annexe)

En évacuant ainsi une partie importante du message français, le traducteur a passablement révisé le contenu du discours d'expression anglaise.

Ce phénomène n'a pas échappé aux observateurs dans les médias. En effet, les journalistes du Québec et du Canada ont rapidement repéré les divergences entre les versions française et anglaise du discours de Lucien Bouchard. Voici trois exemples de la réception, tirés respectivement de La Presse, de The Gazette et du Toronto Star:

(7) Mais M. Bouchard avait un tout autre message, beaucoup moins incisif pour son auditoire anglophone. Alors que le premier ministre a livré la même allocution en français et en anglais, le chef du Bloc québécois a réservé ses flèches contre Jean Chrétien pour sa présentation en français. En anglais, il a plutôt vanté les vertus du projet de partenariat Québec-Canada qu'il entend négocier advenant un OUI. 
(8) Bouchard speaks only in French of panic in Ottawa.

(Wells 1995: A11; voir annexe)

(9) In English, the Bloc leader urged Quebecers to reject the last-minute promises of a man who was a chief architect of the 1982 constitutional deal that Quebec refused to sign and to remember Chretien's role in scuttling the 1990 Meech Lake accord which would have recognized Quebec's distinct nature. [...]

In his French remarks, Bouchard held up a French newspaper headline from 1981 which used a headline [sic] that told the story of how, by approving a constitutional package without Quebec, premier Rene Levesque had been betrayed by then-prime minister Pierre Trudeau and the nine other premiers.

(Vienneau 1995: A1; voir annexe)

On peut dire qu'en général, la presse écrite a davantage porté attention aux écarts présents dans le discours de Lucien Bouchard que dans ceux de Jean Chrétien. S'il est vrai qu'il y avait de grandes différences entre la version française et la version anglaise du discours de Bouchard, peu de journalistes ont remarqué le message «caché» dans la traduction de celui de Chrétien. En effet, certains passages de la version anglaise de Jean Chrétien visaient précisément les nouveaux citoyens reçus du Québec. À preuve le témoignage de Benoît Corbeil, ancien directeur général du PLC (section Québec), paru 10 ans après la tenue du référendum:

C’est clair que la stratégie (du Parti libéral), c'était de faire sortir le vote ethnique au maximum. [...] Jean Chrétien a fait un discours à la nation, un en français et l'autre en anglais, qui n'était pas identique [sic] et qui contenait un message particulier pour les groupes ethniques $^{30}$. (Noël et Marissal 2005: A2)

L'exemple suivant pourrait illustrer ce à quoi faisait référence Benoît Corbeil :

(10) a. Pensez-vous vraiment que vous et votre famille aurez une meilleure protection sociale dans un Québec séparé?

b. Do you really think you and your family will enjoy greater security in a separate Quebec?

(Canada, Premier ministre 1995a: 3; voir annexe; nous soulignons)

Alors que l'anglais met l'accent sur la question de la sécurité, le français évoque la question de la protection sociale, deux sujets qui font contraste. Les francophones connaissaient la tradition pacifique du Québec, tandis que les nouveaux arrivants, qui choisissent souvent l'anglais pour s'intégrer au Québec, provenaient parfois de pays où la démocratie n'est pas bien établie. Ces derniers auraient pu croire au risque de la guerre civile après un "OUI».

Rappelons aussi que le 31 août $1995^{31}$, dans un article du quotidien Toronto Star, on expliquait qu'avant le référendum, le gouvernement fédéral avait procédé à une période intense d'attribution de la citoyenneté au Québec. Des juges avaient dû faire des heures supplémentaires afin que des immigrants qualifiés puissent obtenir leur citoyenneté à temps pour le vote. Il est clair que dans son discours, Jean Chrétien a tenté de plaire à cette nouvelle population d'électeurs, laissant entendre qu'un vote pour le camp du OUI entraînerait l'instabilité sociale et politique au Québec. En faisant allusion (en anglais) à une crise éventuelle, le premier ministre avait tout particulièrement en tête les nouveaux citoyens reçus, qui provenaient parfois de pays 
en guerre civile. Cependant, pour Mátyás Bánhegyi, auteur d’un article sur la traduction de ce discours de Chrétien en 1995, la version anglaise visait la population autochtone. Il écrit:

[F]or historical reasons (primarily the Oka crisis), [the English-speaking aboriginal Canadians] were not very optimistic about the French community and its attitude towards aboriginals. As these aboriginals were inclined to vote against the French Canadians, they were presumably specifically targeted with the English Referendum Speech through its very simple, non-compound sentences and style. (2008:100)

Même s'il est fort possible que les Amérindiens d'expression anglaise aient constitué un public de choix pour le premier ministre, les témoignages recueillis cidessus semblent indiquer que les nouveaux immigrants formaient une cible plus importante que la population amérindienne.

On constate que les écarts de traduction chez Chrétien sont ponctuels et bien ciblés alors que les écarts chez Bouchard sont de l'ordre de l'adaptation, de la transrévision ou de la réécriture. La stratégie du camp du NON (traduction «traditionnelle») semble avoir été plus efficace, du point de vue de la réception médiatique, que la stratégie du camp du OUI (réécriture d'un point de vue macro). Malgré son expérience à titre de ministre au gouvernement fédéral, Lucien Bouchard a utilisé une stratégie qui s'est retournée contre lui: tous les médias ont fait ressortir le fait que son message divergeait en français et en anglais. Même si le message du premier ministre canadien comportait lui aussi des différences d'une langue à l'autre, son discours a été mieux accueilli dans les journaux.

\subsection{Les discours prononcés au soir du référendum de 1995}

Au soir du référendum de 1995, plusieurs hommes politiques ont livré un discours afin de se prononcer sur le résultat du vote. Les discours de Jean Chrétien, de Daniel Johnson et de Jacques Parizeau ont reçu plus d'attention médiatique que les autres et c'est la raison pour laquelle nous les analysons ici. Le discours de Parizeau, improvisé, n’a été prononcé qu'en français. Aucune traduction n’a été fournie aux médias. Le discours de Chrétien, enregistré au cours de la journée, a été présenté dans les deux langues officielles. Le texte du discours a été envoyé aux médias sous la forme de deux communiqués de presse, l'un en français et l'autre en anglais. Le discours de Johnson a été lu devant des militants. Le discours était majoritairement en français, mais il contenait aussi du texte anglais.

Contrairement à ce qui s'était passé quelques jours auparavant, la presse n’a pas traité la question de l'équivalence des deux versions linguistiques du discours de Chrétien. À notre connaissance, seul le quotidien Le Devoir a écrit que le discours avait été prononcé en français et en anglais:

(11) S’adressant aux citoyens d'un océan à l'autre, tour à tour en français et en anglais, M. Chrétien a indiqué que les démonstrations d'affection auxquelles se sont livrés les Canadiens hors Québec dans les dernières heures de la campagne référendaire avaient contribué au moins «en partie» au résultat.

(Dion 2005: A1)

En fait, le discours de Jean Chrétien a été peu critiqué par les médias du pays. Les journalistes ont surtout paraphrasé des pans du discours, sans en faire une analyse 
approfondie. Puisque le discours n'a été que peu critiqué, on peut dire qu'il a été relativement bien reçu.

Le discours de Chrétien comportait de petites divergences d'une version linguistique à l'autre. Précisons que bien souvent, dans les messages prononcés en situation de crise, le premier ministre du Canada ne met pas nécessairement l'accent sur l'identité canadienne dans la version française de ses discours (Gagnon 2006a; 2009). Les exemples de la section précédente nous en ont donné une illustration (voir exemples (2) et (3)). Cependant, au soir du référendum de 1995, Chrétien triomphe, car il a gagné la bataille. Jusqu'à un certain point, la traduction du discours reflète cette victoire.

(12) a. Encore une fois, nous avons montré au monde entier les grandes valeurs de tolérance, d'ouverture et de respect mutuel qui animent notre pays, le Canada.

b. Once again, we have shown the entire world our country's great values of tolerance, openness and mutual respect.

(Canada, Premier ministre 1995b : 1; voir annexe; nous soulignons)

En français, Chrétien tente de mettre en valeur le discours identitaire canadien à l'aide d'une apposition. Grâce à ce procédé, l'image canadienne est plus forte en français. Selon nos recherches, il s'agit d'une occurrence rare pour un discours fédéraliste canadien: il existe des discours télévisés où les deux versions linguistiques mettent l'accent sur l'identité canadienne en parts égales, mais il existe peu d'autres exemples où la version française présente une image canadienne plus forte que la version anglaise. Comme nous l'avons vu, ce type de stratégie de traduction (la valorisation de l'identité canadienne en français) ne semble pas avoir indisposé le lectorat de 1995.

Jusqu'à présent, nous avons observé des stratégies de traduction où une certaine forme de traduction institutionnalisée avait sa place. En d'autres termes, le Bloc Québécois et le Parti libéral du Canada ont fort probablement fait appel à des experts pour travailler sur la version anglaise de leur(s) discours. Dans le cas du Parti Québécois et du discours de son chef Parizeau, nous sommes en présence d'une stratégie de non-traduction. C'est donc que le parti a laissé le soin de la traduction aux médias d'expression anglaise. Observons la traduction du quotidien The Gazette, le journal d'expression anglaise de la communauté montréalaise:

(13) a. C'est vrai, c'est vrai. C'est vrai qu'on a été battus au fond par quoi? Par l'argent puis des votes ethniques, essentiellement.

(Parizeau 1995: B3; voir annexe)

b. We are beaten, it's true. But by what? Money and ethnic vote.

(Authier et Scott 1995: B1, B7; voir annexe)

Lorsqu'on compare l'original français avec la traduction journalistique, on remarque qu'il existe une légère différence dans la façon dont Parizeau a présenté ses affirmations en anglais.

La majorité des médias, tant francophones qu’anglophones, ont critiqué les propos (maladroits) du premier ministre. Cependant, dans la version anglaise, le texte donne l'impression que l'allocution du premier ministre était planifiée, qu'il n'y avait eu aucune hésitation. L'aspect direct, c'est-à-dire non négocié des propos de Parizeau, 
ajoute une touche d'intransigeance qui est moins présente en français. En français, la répétition ("c'est vrai, c'est vrai») et la présence d'un adverbe ("essentiellement») viennent, dans une certaine mesure, amoindrir l'énoncé. Il s'agit d'une stratégie de politesse de la part de Parizeau. Cette stratégie est évacuée de l’anglais.

La traduction de The Globe and Mail pousse encore plus loin cette idée de discours planifié:

(14) a. C'est vrai, c'est vrai qu'on a été battus, au fond par quoi? Par l'argent puis des votes ethniques, essentiellement.

(Parizeau 1995: B3; voir annexe)

b. It is true that we were beaten by the power of money and the ethnic vote.

(Picard 1995: A1-A2; voir annexe)

En anglais, la phrase de Parizeau semble appartenir à un discours rédigé, pas à une allocution spontanée. Dans l'un de ses articles, Brian Mossop (1990) expliquait l'importance de respecter la «voix» d'un premier ministre du Québec lorsqu'il est traduit vers l'anglais. Pour Mossop, les traductions homogénéisantes ont des répercussions sur l'image qu'ont les lecteurs anglophones du premier ministre québécois.

Une dernière chose à propos de la traduction journalistique: il y avait un écart important dans les traductions anglaises du discours de Parizeau.

(15) a. C'est vrai, c'est vrai qu'on a été battus, au fond par quoi? Par l'argent puis des votes ethniques, essentiellement.

(Parizeau 1995: B3; voir annexe; nous soulignons)

b. We are beaten, it's true. But by what? Money and ethnic vote.

(Authier et Scott 1995: B1, B7; voir annexe; nous soulignons)

c. It is true that we were beaten by the power of money and the ethnic vote.

(Picard 1995: A1-A2; voir annexe; nous soulignons)

Il est difficile d'établir avec certitude s'il s'agit d'une erreur ou d'un écart délibéré. Trois arguments viennent appuyer l'hypothèse de l'erreur. Tout d'abord, comme le rappelle Brian Mossop (1990), les erreurs sont fréquentes dans les traductions de journalistes, notamment parce que ces derniers n'ont pas de formation en traduction. Ensuite, les écarts de traduction figurent dans des articles plutôt que dans des éditoriaux. L'éthique du journaliste voudrait que le texte reprenne le plus exactement possible les mots du premier ministre, particulièrement lors de la rédaction d'un compte rendu. Enfin, des journalistes d'expression française ont également mal cité les paroles de Parizeau, évoquant «le vote ethnique» plutôt que "des votes ethniques». Les textes où l'écart apparaît ont donc été rédigés par des journalistes d'horizons différents, ce qui fait penser qu'il n'y a pas eu de traduction concertée.

L'écart textuel de l'exemple (15) accentue le caractère raciste des propos de Parizeau: l'anglais sous-entend que toutes les ethnies ont voté «NON», alors que Parizeau affirme que certaines minorités ethniques ont voté «NON». Cet extrait d'un écrit de Parizeau paru deux ans après le référendum nous éclaire sur cette question:

Je demeure convaincu que le seul critère important quant à l'orientation du vote sur la souveraineté, c'est la langue. Ce n'est ni la race ni la couleur; c'est la langue. Je connais beaucoup de souverainistes d'origine haitienne alors que je n'en connais aucun chez les Jamaïcains... (Parizeau 1997: 41) 
Le texte de Parizeau fait écho à sa pensée du 30 octobre 1995. Pour Parizeau, la question «ethnique» est plurielle. Les journaux d'expression anglaise n'ont pas transmis cette idée dans leurs écrits au lendemain du référendum.

Ajoutons que dans le journal La Presse du 2 novembre 1995, le discours de Parizeau est présenté en son entier. Toutes les nuances du «dialogue» y sont, hésitations et stratégies de politesse comprises. Or, ce genre de publication était plus difficile à faire en anglais puisqu'il n'existait pas de version officielle de la traduction. En choisissant la voie de la non-traduction, Parizeau et son parti ont contribué, dans une certaine mesure, à creuser l'écart qui existe entre le parti et les minorités culturelles québécoises.

Quant au discours de Daniel Johnson, il n’a pas été traduit en entier par le Parti libéral du Québec. En fait, le chef du camp du NON a prononcé un discours bilingue, soit l'essentiel en français, avec quelques phrases en anglais. L'absence d'une traduction officielle complète se confirme à la lecture d'articles de The Globe and Mail et du Toronto Star, qui ont offert des versions différentes des mots d'ouverture du chef de l'opposition:

(16) a. Je tiens à m’adresser à tous les Québécois [...].

(CBC 1995; voir annexe)

b. I want to speak to all Quebecers [...].

(Mackie 1995: A9; voir annexe)

c. I am speaking to all Quebeckers [...].

(Thompson 1995: A9; voir annexe)

En anglais, le temps de verbe diffère d'une version à l'autre, ainsi que l'orthographe du gentilé «Quebecker». S’il existait une version anglaise officielle du texte de Johnson, on ne trouverait pas autant de différences dans cette phrase. Ces écarts ne créent pas nécessairement d'effet d'ordre politique, mais ils établissent que des journalistes ont fort probablement effectué les traductions du texte anglais.

Dans certains cas, les écarts de traduction entre les versions française et anglaise ont des répercussions sur le sens du message envoyé. En voici un exemple:

(17) a. Je leur dis il ne faut pas abandonner ce soir, je leur dis: nous pouvons ensemble continuer à connaître les succès et les progrès qui font le Québec d'aujourd'hui et qui préparent le Québec de demain.

(CBC 1995; voir annexe)

b. But he urged them not to despair because the referendum result «gives Quebeckers the opportunity to build new successes».

(Mackie 1995: A9; voir annexe)

c. I say to them: Don't lose hope. I say to them that together we can continue to achieve progress.

(Thompson 1995: A9; voir annexe)

Dans les extraits en anglais, non seulement la partie sur le «Québec d'aujourd'hui et de demain» a été complètement omise, mais le journaliste de The Globe and Mail a choisi de traduire "succès et progrès " par «successes", alors que le journaliste du Toronto Star a choisi «progress» seulement. Il en résulte que les nuances du message de Johnson sont éliminées en anglais, ce qui rend le discours plus direct, plus tranché. 
Or, Johnson a célébré sa victoire dans la retenue et la nuance, puisque la marge entre le vote victorieux et le vote perdant était très mince. Dans la presse d'expression anglaise, cette caractéristique importante s'estompe.

Même si le PLQ a fourni des extraits en anglais de son discours pour son auditoire anglophone, les journalistes de la presse écrite ont puisé abondamment dans la version française pour leur compte rendu. On en conclut que l'extrait anglais du discours de Johnson ne visait pas la presse écrite, mais plutôt le bulletin d'information télévisé. Autrement, le PLQ aurait fait traduire tout son discours en anglais. Le discours bilingue de Daniel Johnson répond en fait aux exigences télévisuelles canadiennes: comme l'a démontré Kyle Conway (2011: 70), pour renseigner un auditoire à propos de l'autre communauté linguistique, les bulletins télévisés comme The National utilisent fréquemment les propos d'intervenants s'exprimant dans leur langue seconde. Cette méthode est préférée à d'autres techniques langagières, comme la traduction en voix hors champ, la traduction en sous-titres ou le résumé interlingual.

Avec les exemples de Johnson et de Parizeau, nous avons vu que les traductions journalistiques sont parfois erronées ou qu'elles présentent des variations subtiles de l'original, qui entraînent des effets non désirés. L'absence d'un texte officiel anglais semble par ailleurs donner une grande latitude aux journalistes, qui traduisent et paraphrasent les propos des politiciens d'expression française.

\section{Conclusion}

Les résultats présentés ici montrent que la présence ou l'absence d'une stratégie politique de traduction officielle a des répercussions importantes sur la réception des discours. Par exemple, contrairement à la traduction du discours de Jean Chrétien, celle du discours de Bouchard était trop éloignée de ce à quoi s'attendaient les médias pour être bien acceptée. N'oublions pas qu'en matière de traduction des discours politiques, le Parti libéral du Canada a plus d'expérience que le Bloc Québécois, comme l'a notamment démontré l'étude des sites Web de ces deux partis à la section 2 .

On constate par ailleurs que les normes canadiennes en matière de traduction de discours politiques sont intimement associées à la traduction traditionnelle, qui exclut généralement la transrévision et la réécriture. On pourrait affirmer que le PLC a contribué à façonner la norme en matière de traduction des discours politiques: ce parti a été au pouvoir pendant la majeure partie du $\mathrm{xx}^{\mathrm{e}}$ siècle et il a participé activement aux politiques sur le bilinguisme officiel du pays. En étant aussi au fait des normes de traduction au Canada, le PLC a été en mesure de bien faire accepter son discours le 25 octobre 1995, et ce, même s'il y avait quelques écarts importants entre la version française et la version anglaise.

La question de l'absence de traduction officielle a également été abordée dans cet article. Nous avons vu qu'une politique de non-traduction a parfois des effets malencontreux pour le parti qui la pratique. Au Canada et au Québec, les journalistes bilingues effectuent eux-mêmes la traduction des extraits de discours unilingues. Or, ces journalistes-traducteurs ne sont pas à l'abri des erreurs de traduction et leur traduction entraîne parfois une distorsion des stratégies de politesse de l'émetteur. On peut sans doute expliquer le choix stratégique du Parti Québécois en matière de traduction à l'aide de la déclaration de Parizeau dans Pour un Québec souverain (1997), où l'ancien premier ministre établit un lien entre la langue adoptée par un 
immigrant et l'orientation de son vote sur la souveraineté. Cependant, on l'a vu, une telle stratégie nourrit la lutte politique qui existe entre le PQ et les milieux anglophones. Le Parti Québécois est sans doute conscient de cette problématique, puisque pendant une certaine période de temps, son site Web offrait du contenu en anglais. Le retour à l'unilinguisme du site semble indiquer que le multilinguisme (même sommaire) n'a pas répondu aux besoins du parti. Le problème reste donc entier.

Pour le meilleur et pour le pire, la question de la traduction façonne les rapports entre les deux communautés linguistiques du Canada et du Québec. Comme l'a souligné Meylaerts (2011: 743-745), parmi les nombreuses études sur les politiques de langue, le rôle clef de la traduction n'est que peu pris en considération. C'est particulièrement vrai pour le Québec, où la question de la traduction institutionnelle est rarement étudiée. Notre article a pourtant montré que d'un point de vue institutionnel, les répercussions politiques et sociales de la traduction gagneraient à être davantage considérées. De nouvelles recherches sur le sujet sont vivement souhaitables.

\section{REMERCIEMENTS}

L'auteure aimerait exprimer sa reconnaissance au Fonds de recherche sur la société et la culture du Québec, qui a contribué à l'aboutissement du projet. Elle voudrait aussi remercier les deux lecteurs anonymes qui, par leurs commentaires éclairés, ont beaucoup apporté à cet article. Les relectures minutieuses de Gillian Lane-Mercier, de Denise Merkle et de Stéphanie Roesler ont également été fort utiles.

\section{NOTES}

1. Eustace, Chris (2012): Réflexions au sujet de "Lisée gets a seat at the cabinet table... and the barber shop ». Le blogue de Jean-François Lisée (23 septembre 2012). Consulté le 13 août 2014, http:// jflisee.org/lisee-gets-a-seat-at-the-cabinet-table-and-the-barber-shop/\#comments

2. Bourgault-Côté, Guillaume (21 septembre 2012): Anglos: le choix de Lisée fait jaser. La communauté applaudit l'idée d'un messager... mais pas le messager. Le Devoir. 103(215):A1.

3. Macpherson, Don (20 septembre 2012): Marois's choice of bridge builder. The Gazette. A19.

4. Morin-Rossignol, Rino (26 septembre 2012): «Faites-leur sentir». Acadie nouvelle. 7657: 17.

5. Nepveu, Pierre (22 septembre 2012): Langue - Au-delà du français menacé. Le Devoir. 103(216):B5.

6. Finances et Économie Québec (2012): Previous Budgets. Québec: Gouvernement du Québec. Consulté le 13 août 2014, www.budget.finances.gouv.qc.ca/index_en.asp

7. Internet Archive (s.d.) : About the Internet Archive. San Francisco. Consulté le 13 août 2014, http:// archive.org/about/

8. Internet Archive (s.d.): Consulté le 13 août 2014, http://web.archive.org/web/*/http://www.liberal.ca

9. Internet Archive (s.d.) : Consulté le 13 août 2014, http://web.archive.org/web/*/http://www.blocquebecois.org

10. Internet Archive (s.d.): Consulté le 13 août 2014, http://web.archive.org/web/*/http://www.pq.org

11. Internet Archive (s.d.): Consulté le 13 août 2014, http://web.archive.org/web/*/http://www.plq.org/

12. Reproduction autorisée le 26 février 2014 par Caroline Dupré, du Département de graphisme du Parti Québécois.

13. GAGNON, Lysiane (22 septembre 2012): Mme Marois et les anglos. La Presse. 128(278):A39.

14. Héвert, Chantal (4 juin 2012): French endures on the Hill. Toronto Star. A7.

15. Reproduction autorisée le 21 juin 2013 par Catherine Lacroix, adjointe à la direction générale du Bloc Québécois.

16. BibliothèQue du Parlement (2014): Parlement du Canada. Consulté le 13 août 2014, www.parl. gc.ca/parlinfo/Lists/Party.aspx ?Language $=F$

17. École de politique appliquée de l'Université de Sherbrooke (2013): Brève présentation des principaux partis - Canada. In Jean-Herman Guay (dir.), Perspective monde. Consulté le 24 juin 2013, http://perspective.usherbrooke.ca/bilan/servlet/BMListePartisOrientation?codePays=CAN 
18. Giroux, Raymond (4 mai 2011): Le verdict. Ignatieff part, la succession s'amène. Le Soleil. 115(125):7.

19. Bellavance, Joël-Denis (2 mars 2006): Succession de Paul Martin. Le bilinguisme sème la bisbille au PLC. La Presse. 122(130):A10.

20. Reproduction autorisée le 12 juillet 2013 par John J. Arnold, directeur principal, Conformité et administration du Parti libéral du Canada.

21. Reproduction autorisée le 12 juillet 2013 par John J. Arnold, directeur principal, Conformité et administration du Parti libéral du Canada.

22. Nicoud, Anabelle (27 août 2012): Le blues des anglos. La Presse. 128(256):A6.

23. Ici Radio-Canada (2007): Le parti des anglophones? Page consultée le 13 août 2014, http://ici. radio-canada.ca/nouvelles/Politique/2007/04/24/006-plc-parti-anglais.shtml

24. Reproduction autorisée le 18 octobre 2014 par Maxime Roy, directeur des communications au Parti libéral du Québec.

25. Reproduction autorisée le 18 octobre 2014 par Maxime Roy, directeur des communications au Parti libéral du Québec.

26. Michel Bélisle ( $1^{\text {er }}$ août 2002): communication personnelle, courriel.

27. Nos sincères remerciements à Reine Meylaerts, qui nous a envoyé ces renseignements.

28. Publications - Fédération Wallonie-Bruxelles (2005): La Flandre, à travers les dossiers thématiques. Facilités linguistiques. Page consultée le 13 août 2014, www.pfwb.be/le-travail-du-parlement/ doc-et-pub/publications/FRCirculairePeeters4Keulen.pdf

29. CBC/Radio-Canada (1995): Politique des programmes. Page consultée le 13 août 2014, www.cbc. radio-canada.ca/fr/rendre-des-comptes-aux-canadiens/lois-et-politiques/programmation/politiquedes-programmes/1-1-27/

30. NoËL, André et MARISSAL, Vincent (21 avril 2005): Parizeau avait raison, affirme Benoît Corbeil. La Presse. 121(178):A2.

31. Toronto StaR (31 août 1995): Citizenship blitz in Quebec. A2.

\section{RÉFÉRENCES}

Amireault, Valérie (2004): Les immigrants au Québec: le français est de plus en plus parlé à la maison. Québec français. 132: 58-59.

BÁnhegyi, Mátyás (2008): A Translation Studies Oriented Integrative Approach to Canadian Political Discourse. Across Languages and Cultures. 9(1):77-107.

Chesterman, Andrew (1997): Memes of Translation: The Spread of Ideas in Translation Theory. Amsterdam/Philadelphia: John Benjamins.

Conway, Kyle (2011): Everyone Says No. Public Service Broadcasting and the Failure of Translation. Montréal: McGill-Queen's University Press.

De Coster, Michel (2007): Les enjeux des conflits linguistiques: Le français à l'épreuve des modèles belge, suisse et canadien. Paris: L'Harmattan.

Gagnon, Chantal (2006a): Ideologies in the history of translation: A case study on Canadian political speeches. In: Georges BASTIN et Paul BANDIA (dir.), Charting the Future of Translation Studies. Ottawa: Les Presses de l'Université d'Ottawa, 201-223.

Gagnon, Chantal (2006b): Language plurality as power struggle, or: Translating politics in Canada. In: Reine Meylaerts (dir.), Heterolingualism in/and Translation. Target. 18(1):6990.

Gagnon, Chantal (2009): La traduction des discours politiques au Canada. Thèse de doctorat non publiée. Birmingham: Aston University.

GAGnon, Chantal (2012): La visibilité de la traduction au Canada en journalisme politique: mythe ou réalité? Meta. 57(4):943-959.

Gagnon, Chantal (2013): Traduction et politique canadienne: quel est le rôle des journalistes entre 1942 et 1995? Meta. 58(3):522-541.

Godin, Pierre (2001): René Lévesque, l'espoir et le chagrin (1976-1980). Montréal: Boréal.

LiséE, Jean-François (1990): Dans l'œil de l'aigle: Washington face au Québec. Montréal: Boréal.

Meylaerts, Reine (2009): «Et pour les Flamands, la même chose» : quelle politique de traduction pour quelles minorités linguistiques? Meta. 54(1):7-21. 
Meylaerts, Reine (2011): Translational Justice in a Multilingual World: An Overview of Translational Regimes. Meta. 56(4):743-757.

Mossop, Brian (1990): Translating Institution and «Idiomatic» Translations. Meta. 35(2):342354.

Parizeau, Jacques (1997): Pour un Québec souverain. Montréal: VLB.

Piroth, Scott (2012): A Bilingual Legislature? Question Period in Canada's House of Commons. The Journal of Legislative Studies. 18(2):161-183.

\section{ANNEXE: inventaire du corpus}

Authier, Philipp et Scott, Sarah (31 octobre 1995) : Parizeau: Most francophones vote Yes. The Gazette. B1, B7.

Canada, Premier ministre (1995a): Address to the nation by Prime Minister Jean Chrétien, October 25, 1995 = Message à la nation du premier ministre Jean Chrétien, le 25 octobre 1995. Ottawa: Cabinet du premier ministre.

Canada, Premier ministre (1995b): Statement by Prime Minister Jean Chrétien, Ottawa, October 30, 1995 = Déclaration du Premier ministre Jean Chrétien, Ottawa, le 30 octobre 1995. Ottawa: Cabinet du premier ministre.

CBC (1995): The 1995 Quebec Referendum: Daniel Johnson's Victory Speech. Consulté le 26 mars 2014, www.cbc.ca/archives/categories/politics/federal-politics/separation-anxiety-the1995-quebec-referendum/daniel-johnsons-victory-speech.html

Dion, Jean (31 août 1995): Chrétien: Au Canada de ne pas laisser tomber les Québécois. Le Devoir. A1.

HÉBert, Chantal (26 octobre 1995): Ultime appel. La Presse. 127(7):A2.

Le Point. Radio-Canada (25 octobre 1995): Un oui devient possible. Consulté le 26 juin 2013 , $<$ http://archives.radio-canada.ca/politique/provincial_territorial/clips/12187/>

MACKIE, Richard (31 octobre 1995): THE REFERENDUM No side holds rest of Canada to promise Constitutional change needed, federalists say, as Johnson's nightmare of divided Quebec comes true. The Globe and Mail. 152:A9.

PARIzeAu, Jacques (2 novembre 1995): On a été battus par l'argent et par les votes ethniques! La Presse. B3.

PICARD, André (31 octobre 1995b) : NO - by a whisker. Parizeau promises to «exact revenge» or sovereigntist loss. The Globe and Mail. A1-A2.

THE GLOBE AND MAIL (26 octobre 1995a): The Referendum. Addressing the nation. 152:A16.

The Globe AND MaIL (26 octobre 1995b): Prime Minister can't be trusted, Bouchard says. 152:A16.

Thompson, Allan (31 octobre 1995): Johnson offers reconciliation to Yes side. The Toronto Star. A9.

Vienneau, David (26 octobre 1995): PM pitches to undecided "Dangerous risk" in Yes vote, Chretien says. The Toronto Star. A1.

Wells, Paul (26 octobre 1995): Canada the best: PM. But Bouchard sees two solitudes. The Gazette. A1, A11. 